\title{
FURTHER RESULTS ON POSITIVE PERIODIC SOLUTIONS OF IMPULSIVE FUNCTIONAL DIFFERENTIAL EQUATIONS AND APPLICATIONS
}

\author{
YUJI LIU ${ }^{1}$
}

(Received 5 March, 2007; revised 28 September, 2008)

\begin{abstract}
A class of first-order impulsive functional differential equations with forcing terms is considered. It is shown that, under certain assumptions, there exist positive $T$-periodic solutions, and under some other assumptions, there exists no positive $T$-periodic solution. Applications and examples are given to illustrate the main results.

2000 Mathematics subject classification: primary 34B10; secondary 34B15, 34K15, 34K10, 34C25, 92D25.

Keywords and phrases: impulse, first-order functional differential equation, positive $T$-periodic solution, fixed point theorem.
\end{abstract}

\section{Introduction}

Impulsive functional differential equations, which are functional differential equations involving impulse effects, appear as a natural description of observed evolution phenomena of several real-world problems subject to a short-term perturbation whose duration is negligible in comparison with the duration of the process [4, 6-9, 43, 46, 48, 54]. The interest of researchers in this field has grown very fast due to applications to real-world phenomena and impulsive functional equations have been analyzed by many authors in the literature (see [2, 14-22, 24-26, 28, 31, 33, $37,38,40,44,49-51]$ and references therein). The existence of periodic solutions or positive periodic solutions of impulsive functional differential equations has become an active research area in this field.

Supported by the Natural Science Foundation of Hunan province, People's Republic of China (No 06JJ5008) and Natural Science Foundation of Guangdong province (No 7004569).

${ }^{1}$ Department of Mathematics, Guangdong University of Business Studies, Guangzhou 510320,

People's Republic of China; e-mail: liuyuji888@sohu.com.

(C) Australian Mathematical Society 2009, Serial-fee code 1446-1811/2009\$16.00 
In this paper, we consider the existence and nonexistence of positive periodic solutions of the following first-order functional differential equation

$$
x^{\prime}(t)=-a(t) x(t)-\lambda h(t) f(t, x(t-\tau(t, x(t))))+r(t), \quad t \in R, \quad t \neq t_{k}, k \in Z
$$

with the impulse effects

$$
x\left(t_{k}\right)=\left(1+t_{k}\right) x\left(t_{k}^{-}\right), \quad k \in Z,
$$

where:

(i) $T>0$ is a constant, $\lambda>0$ is a parameter, $Z$ is the set of all integers and $R$ is the set of all real numbers;

(ii) $\left\{t_{k}\right\}$ is a sequence such that there exists $l>0$ with $t_{k}+T=t_{k+l}$ for all $k \in Z$, $b_{k}>-1$ constants for all $k \in Z$;

(iii) $a: R \rightarrow R, h: R \rightarrow R$ and $r: R \rightarrow R$ are $T$-periodic functions in the variable $t$;

(iv) the functions $\tau: R \times R \rightarrow[0,+\infty)$ and $f: R \times R \rightarrow R$ are $T$-periodic in $t$ and continuous in $x$.

The purpose of this paper is to establish new existence and nonexistence criteria for positive $T$-periodic solutions of (1.1) with impulse effects (1.2) (System (1.1)-(1.2) for short). Our results are different from those in [17, 19, 22, 29, 31, 45, 50] since $a$ and $f$ are allowed to change sign, and there is a forcing term in (1.1). The methods used in this paper are based on Green's functions and fixed point theorems in cones in Banach spaces and the techniques are different from known versions. When the main results are applied to some impulsive biological models, the new results, which are different from known results in [49, 51], are derived for positive $T$-periodic solutions of these models.

The study of positive $T$-periodic solutions of System (1.1)-(1.2) is mainly motivated by the publications of the following interesting results.

First, (1.1) is called a state-dependent functional differential equation and has many applications. It can be interpreted as the standard Malthus population model $x^{\prime}(t)=-a(t) x(t)$ subject to the perturbation $-\lambda h(t) f(t, x(t-\tau(t, x(t))))$ and the forcing term $r(t), \tau(t, x(t))$ is called a state-dependent variable which was first posed by Hale and Lonel, see [10, 13, 14] and the references therein. The existence of periodic solutions of state-dependent functional differential equations was studied in $[1,23,30,45]$ and the references therein. Impulse effects (1.2) are a linear case, which occur at a $T$-periodic fixed moment of time and are a simple case of impulsive functions, see [27-29, 44] and the references therein.

System (1.1)-(1.2) contains the following well-known impulsive biological models as special cases:

$$
\begin{cases}N^{\prime}(t)=-\mu(t) N(t)+\lambda p(t) e^{-r(t) N(t-\tau(t))}, & t \neq t_{k}, \quad k \in Z, \\ N\left(t_{k}\right)=\left(1+b_{k}\right) N\left(t_{k}^{-}\right), & k \in Z,\end{cases}
$$




$$
\begin{cases}N^{\prime}(t)=-\mu(t) N(t)+\lambda p(t) \frac{1}{1+r(t) N(t-\tau(t))}, & t \neq t_{k}, k \in Z, \\ N\left(t_{k}\right)=\left(1+b_{k}\right) N\left(t_{k}^{-}\right), & k \in Z,\end{cases}
$$

and

$$
\begin{cases}N^{\prime}(t)=-\mu(t) N(t)+\lambda p(t) N(t-\tau(t)) e^{-r(t) N(t-\tau(t))}, & t \neq t_{k}, k \in Z, \\ N\left(t_{k}\right)=\left(1+b_{k}\right) N\left(t_{k}^{-}\right), & k \in Z,\end{cases}
$$

where $\mu, p, r$ and $\tau$ are positive $T$-periodic continuous functions, $n>0$ a real number, $\lambda>0$ a parameter and $b_{k}>-1 ; N(t)$ denotes the size of the biological population at time $t$.

Equation (1.3) is called the impulsive model of the red blood cell system; the red blood cell model was introduced in [47] and studied in [34]. The existence and attractivity of positive $T$-periodic solutions of some special cases of (1.3) were studied in [49] and [51].

Equation (1.4) is called the impulsive hematopoiesis model; the hematopoiesis model was posed in [48]. The authors in [32] and [41] studied the existence and attractivity of positive $T$-periodic solutions of the following hematopoiesis model without impulse effects

$$
N^{\prime}(t)=-a(t) N(t)+\sum_{i=1}^{m} p_{i}(t) \frac{b_{i}(t)}{1+N^{n}\left(t-\tau_{i}(t)\right)} .
$$

Equation (1.5) is called the impulsive Nicholson's blowfly model; Nicholson's blowfly model was introduced in [9, 39]. Recently, the authors in [42] studied the oscillatory and global attractivity in a periodic Nicholson's blowfly model without impulse effects. Li and Wang [21] investigated the existence and global attractivity of positive periodic solutions for a class of impulsive delay Nicholson's blowfly models. We note that in all of the above-mentioned papers, $\mu, p, r$ are assumed to be nonnegative, but $\mu, p, r$ may change sign by their biological significance. A problem appears naturally.

P1. What conditions guarantee the existence of positive $T$-periodic solutions of (1.3), (1.4) and (1.5), respectively, when $\mu$ and $r$ change sign?

Second, for impulsive functional differential equations, in [16], Li and Huo obtained sufficient and realistic conditions for the existence and global attractivity of positive $T$-periodic solutions of the delay differential system with impulses

$$
\begin{cases}y^{\prime}(t)=y(t) f\left(t, y\left(t-\tau_{1}(t)\right), \ldots, y\left(t-\tau_{m}(t)\right)\right), & t \in R, t \neq t_{k}, k \in Z, \\ y\left(t_{k}^{+}\right)=\left(1+b_{k}\right) y\left(t_{k}\right), & k \in Z .\end{cases}
$$

The method used in [16] involves applying Gaines and Mawhin's coincidence degree theory, constructing suitable Lyapunov functionals and estimating uniform upper bounds on solutions. The results in [16] are applied to some special delay population models. 
Consider the impulse effects. Recently, the authors of [26] studied the system

$$
\begin{cases}x^{\prime}(t)+a(t) x(t)=\lambda h(t) f(x(t-\tau(t))), & t \in R, t \neq t_{k}, k \in Z, \\ x\left(t_{k}^{+}\right)=\left(1+b_{k}\right) x\left(t_{k}\right), & k \in Z .\end{cases}
$$

where $b_{k}>-1$ for all $k \in Z, \lambda>0, a, h$ are $T$-periodic functions. It was proved that System (1.6) has at least one positive $T$-periodic solution under the following assumptions:

(H1) $f$ is nonnegative and continuous with $f(0)>0, h$ is continuous and $T$-periodic and there exists a constant $k>1$ such that

$$
\int_{t}^{t+T} G(t, s) h^{+}(s) d s \geq \int_{t}^{t+T} G(t, s) h^{-}(s) d s \quad \text { for all } t \in R,
$$

where $h^{+}(t)=\max \{h(t), 0\}$ and $h^{-}(t)=\min \{0, h-h(t)\}$ and

$$
G(t, s)=\frac{\exp \left(\int_{t}^{s} a(u) d u\right) \prod_{s<t_{k} \leq t+T}\left(1+b_{k}\right)}{\exp \left(\int_{0}^{T} a(u) d u\right)-\prod_{0<t_{k} \leq T}\left(1+b_{k}\right)} ;
$$

(H2) $a$ is continuous and $T$-periodic and satisfies

$$
\exp \left(\int_{0}^{T} a(s) d s\right)>\prod_{0<t_{k} \leq T}\left(1+b_{k}\right) .
$$

In [19], Li and Shen proved existence results for positive $T$-periodic solutions of (1.6) by using a fixed-point theorem on cones in Banach spaces. In [31], the authors studied the System (1.6) and established the existence and nonexistence of positive periodic solutions of (1.6). The results show us that impulses may cause the existence of positive periodic solutions. The methods employed in [31] are a fixed-point index theorem, Leray-Schauder degree and upper and lower solutions.

In [50], Yan employed a fixed-point index theorem to study the existence and nonexistence of positive periodic solutions for the periodic impulsive functional differential equations with two parameters similar to (1.6). Several existence and nonexistence results are established.

In [22], Li et al. were concerned with the existence of positive periodic solutions to (1.6). The approach is based on the fixed-point theorem in cones. In [34], the nonnegativity of the nonlinear function $f$ is assumed.

In [17], Li et al. discussed the existence of single and multiple positive periodic solutions to nonautonomous functional differential equations with impulse actions at fixed moments similar to (1.6). By employing a fixed-point theorem in cones, based on the nonnegativity of the nonlinear function $f$, the authors established multiple existence results. 
In [29], Liu and Ge studied the existence of positive $T$-periodic solutions of the equation

$$
\begin{cases}x^{\prime}(t)=-a(t) x(t)+\lambda h(t) f(t, x(t-\tau(t, x(t))))+r(t), & t \neq t_{k}, k \in Z, \\ x\left(t_{k}^{+}\right)=\left(1+b_{k}\right) x\left(t_{k}\right), & k \in Z\end{cases}
$$

under the assumptions

$$
\exp \left(\int_{0}^{T} a(s) d s\right)>\prod_{0<t_{k} \leq T}\left(1+b_{k}\right)
$$

or

$$
\exp \left(\int_{0}^{T} a(s) d s\right)=\prod_{0<t_{k} \leq T}\left(1+b_{k}\right)
$$

P2. What conditions govern the existence of positive $T$-periodic solutions of (1.7) with $a, h$ or $f$ changing sign or $\exp \left(\int_{0}^{T} a(s) d s\right)<\prod_{0<t_{k} \leq T}\left(1+b_{k}\right)$ ?

There has been a large number of papers concerned with the existence of solutions of periodic boundary-value problems for first-order functional differential equations with impulse effects. For example, the solvability of the system

$$
\begin{cases}x^{\prime}(t)+a(t) x(t)=f(t, x(t)), & t \in[0, T] \backslash\left\{t_{1}, \ldots, t_{p}\right\} \\ x\left(t_{k}^{+}\right)-x\left(t_{k}\right)=I_{k}\left(x\left(t_{k}\right)\right), & k=1,2, \ldots, t_{p} \\ x(0)=x(T) & \end{cases}
$$

was studied in $[2,5,18,35]$ and the references therein. The methods used in these papers are based on the Shaeffer's fixed-point theorem.

In [5, 11, 18, 24, 36, 38], using lower and upper solution methods, the authors studied the solvability of periodic boundary value problems of first-order functional differential equations with impulses effects. Existence results for solutions are established.

Recently, by using Mawhin's continuation theorem and Shaeffer's fixed-point theorem, the authors of [25] studied the existence of solutions of the periodic boundary value problem for the impulsive functional differential equation

$$
\begin{cases}x^{\prime}(t)+a(t) x(t)=f\left(t, x(t), x\left(\alpha_{1}(t)\right), \ldots, x\left(\alpha_{n}(t)\right)\right), & t \in[0, T] \backslash\left\{t_{1}, \ldots, t_{p}\right\}, \\ x\left(t_{k}^{+}\right)-x\left(t_{k}\right)=I_{k}\left(x\left(t_{k}\right)\right), & k=1,2, \ldots, t_{p}, \\ x(0)=x(T), & \end{cases}
$$

in the cases $\int_{0}^{T} a(s) d s=0, \int_{0}^{T} a(s) d s \neq 0$.

$\mathrm{Li}$ and Shen [20] studied the existence and approximation of solutions for an impulsive delay differential equation with periodic boundary value conditions by 
using comparison principles and monotone iterative techniques. Li and Huo [15] investigated the existence and global attractivity of positive periodic solutions of a class of functional differential equations with impulses.

In this paper we present solutions to problems P1 and P2. Our theorems also generalize and improve known results. The methods used in this paper can be applied to establish existence and nonexistence results of positive solutions of periodic boundary value problems of impulsive functional differential equations.

The remainder of this paper is organized as follows. In Section 2, we give the main results. In Section 3, we apply the main theorems to biological models (1.3), (1.4) and (1.5). Two examples are presented to illustrate the main theorems at the end of this paper.

\section{Main results}

In this section, we present the main results. Choose

$$
X=\left\{\begin{array}{c}
x: R \rightarrow R: x \text { is } T \text {-periodic, continuous on }\left[t_{k}, t_{k+1}\right) \\
\text { there exists the limit } \lim _{t \rightarrow t_{k}^{-}} x(t)=x\left(t_{k}^{-}\right) \text {for all } k \in Z \\
\text { and } x\left(t_{k}\right)=\left(1+b_{k}\right) x\left(t_{k}^{-}\right) \text {for all } k \in Z
\end{array}\right\} .
$$

For $x \in X$, let $\|x\|=\sup _{t \in[0 ; T]}|x(t)|$. It is easy to show that $X$ is a Banach space.

For $a \in X$, we use the notation $a^{+}(t)=\max \{0, a(t)\}$ and $a^{-}(t)=\max \{-a(t), 0\}$. For $b_{k} \in R$, we use the notation $b_{k}^{+}=\max \left\{0, b_{k}\right\}$ and $b_{k}^{-}=\max \left\{-b_{k}, 0\right\}$.

Let us list some assumptions:

(H3) $f: R \times[0,+\infty) \rightarrow R$ satisfies $f(\cdot, x) \in X$ and $f(t, \cdot)$ is continuous and there exists $M>0$ such that $f(t, x)>-M$ for $(t, x) \in R \times R$.

(H3) $f: R \times[0,+\infty) \rightarrow[0,+\infty)$ satisfies $f(\cdot, x) \in X$ and $f(t, \cdot)$ is continuous.

(H4) $a: R \rightarrow R, r: R \rightarrow R, h: R \rightarrow R^{+}$with $a, r, h \in X, \tau(\cdot, x): R \rightarrow R$ are $T$-periodic functions with $\tau(\cdot, x) \in X$, and $\int_{0}^{T} h(s) d s>0, \tau(t, \cdot)$ is continuous.

(H5) $\lambda>0$ is a parameter and $T>0$ is a constant.

(H6) $f(t ; 0)>0$ for all $t \in R$.

(H7) $\lim _{x \rightarrow+\infty}(f(t, x) / x)=N \in(0 ;+\infty]$ uniformly on $[0, T]$.

(H8) $\left\{t_{k}\right\}$ is a sequence for which there exists $l>0$ such that $t_{k}+T=t_{k+l}$ for all $k \in Z, b_{k}>-1$ constants for all $k \in Z$ with $\prod_{t<t_{k} \leq t+T}\left(1+b_{k}\right)=$ constant for all $t \in R$.

(H9) $\exp \left(\int_{0}^{T} a(s) d s\right)<\prod_{0<t_{k} \leq T}\left(1+b_{k}\right)$.

(H10) $r(t) \equiv 0$ for $t \in R$.

(H11) $L$ and $l$ are defined by

$$
\lim _{x \rightarrow+\infty} \sup _{t \in[0, T]} \frac{f(t, x)}{x}=L \in(0,+\infty], \quad \lim _{x \rightarrow 0} \inf _{t \in[0, T]} \frac{f(t, x)}{x}=l \in[0,+\infty) .
$$


(H11) $L^{\prime}$ and $l^{\prime}$ are defined by

$$
\lim _{x \rightarrow+\infty} \inf _{t \in[0, T]} \frac{f(t, x)}{x}=L^{\prime} \in[0,+\infty), \quad \lim _{x \rightarrow 0} \sup _{t \in[0, T]} \frac{f(t, x)}{x}=l^{\prime} \in(0,+\infty] .
$$

To help with the presentation of the main results, we give some lemmas.

Lemma 2.1. Suppose that $a, \sigma_{1} \in X$ and (H8), (H9) hold. If $x \in X$ is a solution of the equation

$$
\begin{cases}x^{\prime}(t)=-a(t) x(t)-\sigma_{1}(t), & t \neq t_{k}, k \in Z \\ x\left(t_{k}\right)=\left(1+b_{k}\right) x\left(t_{k}^{-}\right), & k \in Z\end{cases}
$$

then

$$
x(t)=-\int_{t}^{t+T} G(t, s) \sigma_{1}(s) d s, \quad t \in R, t \neq t_{k},
$$

where $G(t, s)$ is defined as in $(\mathrm{H} 1)$.

PROOF. Since $x \in X$ is a solution of (2.1), we obtain

$$
\left(x(t) e^{\int_{0}^{t} a(u) d u}\right)^{\prime}=-\sigma_{1}(t) e^{\int_{0}^{t} a(u) d u}, \quad t \in R, \quad t \neq t_{k}, k \in Z .
$$

Suppose that $t \in\left[t_{k-1}, t_{k}\right)$. One finds that $t+T \in\left[t_{k-1}+T, t_{k}+T\right)=\left[t_{k-1+l}, t_{k+l}\right)$. Then

$$
\begin{aligned}
x\left(t_{k}^{-}\right) e^{\int_{0}^{t_{k}} a(u) d u}-x(t) e^{\int_{0}^{t} a(u) d u} & =-\int_{t}^{t_{k}} \sigma_{1}(s) e^{\int_{0}^{s} a(u) d u} d s, \\
x\left(t_{i}^{-}\right) e^{\int_{0}^{t_{i}} a(u) d u}-x\left(t_{i-1}\right) e^{\int_{0}^{t_{i}-1} a(u) d u} & =-\int_{t_{i-1}}^{t_{i}} \sigma_{1}(s) e^{\int_{0}^{s} \phi(x(u)) a(u) d u} d s
\end{aligned}
$$

for all $i=k, k+1, \ldots, k-1+l$, and

$$
x(t+T) e^{\int_{0}^{t+T} a(u) d u}-x\left(t_{k-1+l}\right) e^{\int_{0}^{t_{k-1+l}} a(u) d u}=-\int_{t_{k-1+l}}^{t+T} \sigma_{1}(s) e^{\int_{0}^{s} a(u) d u} d s .
$$

Since $x\left(t_{i}\right)=\left(1+b_{i}\right) x\left(t_{i}^{-}\right)$and $x(t)=x(t+T)$, by deleting $x\left(t_{i}^{-}\right)$and $x\left(t_{i}\right)$ we obtain

$$
\begin{gathered}
x(t) e^{\int_{0}^{t+T} a(u) d u}-\prod_{t<t_{k} \leq t+T}\left(1+b_{k}\right) x(t) e^{\int_{0}^{t} a(u) d u} \\
=-\int_{t}^{t+T} \sigma_{1}(s) \prod_{s<t_{k} \leq t+T}\left(1+b_{k}\right) e^{\int_{0}^{s} a(u) d u} d s .
\end{gathered}
$$

It follows that

$$
x(t)=\int_{t}^{t+T} D \sigma_{1}(s) \prod_{s<t_{k} \leq t+T}\left(1+b_{k}\right) e^{\int_{t}^{s} a(u) d u} d s
$$


where

$$
D=\left[-\exp \left(\int_{0}^{T} a(u) d u\right)+\prod_{0<t_{k} \leq T}\left(1+b_{k}\right)\right]^{-1} .
$$

The proof is complete.

LEMma 2.2. Suppose that (H8) and (H9) hold, a, $\sigma_{1} \in X, \sigma_{1}$ is nonnegative and $x \in X$ is a solution of (2.1). Then $x(t) \geq \sigma\|x\|$ for all $t \in R, t \neq t_{k}, k \in Z$, where

$$
\sigma=\frac{\prod_{0<t_{k} \leq T}\left(1-b_{k}^{-}\right)}{\prod_{0<t_{k} \leq T}\left(1+b_{k}^{+}\right)} e^{-\int_{0}^{T}|a(u)| d u} .
$$

Proof. Let $\quad I^{t}=[t, t+T]$, and $I_{1}^{t}=\left\{t \in I^{t} \mid a(t) \geq 0\right\}, I_{2}^{t}=\left\{t \in I^{t} \mid a(t)<0\right\}$. Then (H8) and (H9) imply that

$$
\begin{aligned}
-G(t, s) & =D \exp \left(\int_{t}^{s} a(u) d u\right) \prod_{s<t_{k} \leq t+T}\left(1+b_{k}\right) \\
& \leq D \exp \left(\int_{[t, s] \cap I_{1}^{t}} a(u) d u+\int_{[t, s] \cap I_{2}^{t}} a(u) d u\right) \prod_{s<t_{k} \leq t+T}\left(1+b_{k}^{+}\right) \\
& \leq D \exp \left(\int_{[t, t+T] \cap I_{1}^{t}} a(u) d u\right) \prod_{t<t_{k} \leq t+T}\left(1+b_{k}^{+}\right) \\
& \leq D \exp \left(\int_{0}^{T} a^{+}(u) d u\right) \prod_{0<t_{k} \leq T}\left(1+b_{k}^{+}\right)=M_{1},
\end{aligned}
$$

and

$$
\begin{aligned}
-G(t, s) & \geq D\left[\exp \left(\int_{[t, s] \cap I_{1}^{t}} a(u) d u+\int_{[t, s] \cap I_{2}^{t}} a(u) d u\right) \prod_{s<t_{k} \leq t+T}\left(1-b_{k}^{-}\right)\right] \\
& \geq D \exp \left(\int_{[t, t+T] \cap I_{2}^{t}} a(u) d u\right) \prod_{t<t_{k} \leq t+T}\left(1-b_{k}^{-}\right) \\
& \geq D \exp \left(-\int_{0}^{T} a^{-}(u) d u\right) \prod_{0<t_{k} \leq T}\left(1-b_{k}^{-}\right)=N_{1} .
\end{aligned}
$$

It is easy to see from Lemma 2.1 that

$$
x(t)=-\int_{t}^{t+T} G(t, s) \sigma_{1}(s) d s \geq N_{1} \int_{t}^{t+T} \sigma_{1}(s) d s=N_{1} \int_{0}^{T} \sigma_{1}(s) d s,
$$

and

$$
x(t)=-\int_{t}^{t+T} G(t, s) \sigma_{1}(s) d s \leq M_{1} \int_{t}^{t+T} \sigma_{1}(s) d s=M_{1} \int_{0}^{T} \sigma_{1}(s) d s .
$$


Then

$$
\|x\| \leq M_{1} \int_{0}^{T} \sigma_{1}(s) d s \leq \frac{M_{1}}{N_{1}} x(t)
$$

It follows that

$$
x(t) \geq \frac{N_{1}}{M_{1}}\|x\|=\sigma\|x\|, \quad t \in R
$$

The proof is complete.

Lemma 2.3. Let $X$ be a Banach space and $P \subset X$ a cone of $X$, with $0 \in \Omega_{1} \subset$ $\Omega_{2} \subset X$ open and bounded nonempty subsets. Suppose that $\mathbf{T}: P \cap\left(\overline{\Omega_{2}} \backslash \Omega_{1}\right) \rightarrow P$ is completely continuous. If:

(i) $\|\mathbf{T} x\| \leq\|x\|$ for all $x \in P \partial \Omega_{1},\|\mathbf{T} x\| \geq\|x\|$ for all $x \in P \cap \partial \Omega_{2}$; or

(ii) $\|\mathbf{T} x\| \geq\|x\|$ for all $x \in P \partial \Omega_{1},\|\mathbf{T} x\| \leq\|x\|$ for all $x \in P \cap \partial \Omega_{2}$;

then $\mathbf{T}$ has at least one fixed point $x \in P \cap\left(\overline{\Omega_{2}} \backslash \Omega_{1}\right)$.

Choose

$$
P=\left\{x \in X: x\left(t_{k}\right)=\left(1+b_{k}\right) x\left(t_{k}^{-}\right) \text {for all } k \in Z \text { and } x(t) \geq \sigma\|x\| \text { for all } t \in R\right\} .
$$

It is easy to see that $P$ is a cone in the space $X$. Now, we present main results.

TheOREM 2.4. Suppose that (H3)', (H4), (H5), (H7)-(H9) hold. Then System (1.1)(1.2) has at least one positive $T$-periodic solution if $\lambda \in(A, B]$, where $A$ and $B$ are defined by

$$
\begin{aligned}
& A=2\left[D N \sigma \prod_{0<t_{k} \leq T}\left(1-b_{k}^{-}\right) \exp \left(-\int_{0}^{T} a^{-}(u) d u\right) \int_{0}^{T} h(s) d s\right]^{-1}, \\
& B=R_{0}\left[D M_{2} \sigma \prod_{0<t_{k} \leq T}\left(1+b_{k}^{+}\right) \exp \left(\int_{0}^{T} a^{+}(u) d u\right) \int_{0}^{T} h(s) d s\right]^{-1},
\end{aligned}
$$

and

$$
\begin{aligned}
R(t) & =\int_{t}^{t+T} G(t, s) r(s) d s, \quad R_{0}=\|R\|, \\
g(t, x) & = \begin{cases}f(t, x), & t \in R, \quad x \geq 0, \quad M_{2}= \\
f(t, 0), & t \in R, \quad x<0,\end{cases}
\end{aligned}
$$

PROOF. It is easy to see from (H8) and (H9) that $R(t)$ satisfies

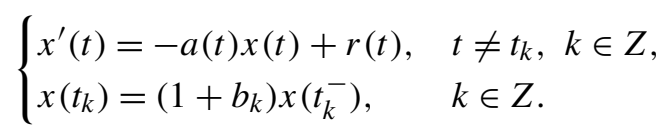


Now, let $\bar{y}(t)=y(t)+R(t)$, consider

$$
\left\{\begin{aligned}
y^{\prime}(t)= & -a(t) y(t)-\lambda h(t) f(t, y(t-\tau(t, \bar{y}(t))) & & \\
& +R(t-\tau(t, \bar{y}(t)))), & & t \neq t_{k}, k \in Z, \\
y\left(t_{k}\right)= & \left(1+b_{k}\right) y\left(t_{k}^{-}\right), & & k \in Z .
\end{aligned}\right.
$$

Then System (1.1)-(1.2) has a positive $T$-periodic solution $x \in X$ if and only if $y(t)=x(t)-R(t)$ is a $T$-periodic solution of (2.2) and $y(t)+R(t)>0$ for all $t \in$ $[0, T]$. Let $\mathbf{T}$ be defined by

$$
\mathbf{T} y(t)=\int_{t}^{t+T} G(t, s) h(s) g(s, y(s-\tau(s, \bar{y}(s)))+R(s-\tau(s, \bar{y}(s)))) d s
$$

for $y \in X$. It is easy to see, from $(\mathrm{H} 3)^{\prime},(\mathrm{H} 4)$, (H8), (H9), that $\mathbf{T}$ is completely continuous and $\mathbf{T} P \subset P$ by the same methods used in Lemma 2.2.

Let $\lambda \in(A, B]$ be fixed. To obtain a positive $T$-periodic solution of System (1.1)(1.2), we perform two steps.

Step 1. Set $\Omega_{1}=\left\{x \in X:\|x\|<R_{0} / \sigma\right\}$. Then one finds, for $y \in P \cap \partial \Omega_{1}$, that

$$
y(t)+R(t) \leq\|y\|+\|R\|=R+0(\sigma+1) / \sigma
$$

and

$$
y(t)+R(t) \geq \sigma\|y\|-\|R\|=0 .
$$

Then

$$
\begin{aligned}
\mathbf{T} y(t) & \leq-\lambda M_{2} \int_{t}^{t+T} G(t, s) h(s) d s \\
& \leq \lambda M_{2} D_{+} E_{+} \quad(\text { using } \lambda \leq B) \\
& \leq \frac{R_{0}}{\sigma}=\|y\|,
\end{aligned}
$$

where

$$
\begin{aligned}
& D_{+}=\left[-\exp \left(\int_{0}^{T} a^{+}(u) d u\right) \prod_{0<t_{k} \leq T}\left(1+b_{k}\right)\right]^{-1} \text { and } \\
& E_{+}=\prod_{0<t_{k} \leq T}\left(1+b_{k}^{+}\right) \exp \left(\int_{0}^{T} a^{+}(u) d u\right) \int_{0}^{T} h(s) d s .
\end{aligned}
$$

Then $\|\mathbf{T} y\| \leq\|y\|$ for all $y \in P \cap \partial \Omega_{1}$.

Step 2. Since $\lambda>A$, we choose $\epsilon>0$ such that

$$
\frac{D_{+}}{2} \lambda(N-\epsilon) E_{-}>\frac{1}{\sigma},
$$

where

$$
E_{-}=\prod_{0<t_{k} \leq T}\left(1-b_{k}^{-}\right) \exp \left(-\int_{0}^{T} a^{-}(u) d u\right) \int_{0}^{T} h(s) d s .
$$


By (H7), one can choose $H>R_{0} / \sigma>R_{0}$ such that

$$
\frac{g(t, y)}{y}=\frac{f(t, y)}{y}>N-\epsilon \text { for } t \in[0, T], y \geq H .
$$

Set $\Omega_{2}=\left\{x \in X:\|x\|<\left(H+R_{0}\right) / \sigma\right\}$. We find, for $y \in P \cap \partial \Omega_{2}$, that

$$
y(t)+R(t) \geq \sigma\|y\|-R_{0} \geq \sigma \frac{H+R_{0}}{\sigma}-R_{0}=H .
$$

Then

$$
\begin{aligned}
\mathbf{T} y(t) & \geq-\lambda \int_{t}^{t+T} G(t, s) h(s)(N-\epsilon)[y(s-\tau(s, \bar{y}(s)))+R(s,-\tau(s, \bar{y}(s)))] d s \\
& \geq-\lambda(N-\epsilon) \int_{t}^{t+T} G(t, s) h(s)\left(\sigma\|y\|-R_{0}\right) d s \\
& \geq \lambda H(N-\epsilon) D_{+} E_{-} \geq \lambda \frac{H+R_{0}}{2}(N-\epsilon) D_{+} E_{-} \geq \frac{H+R_{0}}{\sigma}=\|y\| .
\end{aligned}
$$

Then $\|\mathbf{T} y\| \geq\|y\|$ for $y \in P \cap \partial \Omega_{2}$. Hence, $\mathbf{T}$ has at least one fixed point $y$ such that $R_{0} / \sigma \leq\|y\| \leq\left(H+R_{0}\right) / \sigma$. So $y(t)$ is a positive $T$-periodic solution of (2.2).

On the other hand,

$$
y(t)+R(t)>\sigma\|y\|-R_{0}>\sigma \frac{R_{0}}{\sigma}-R_{0}=0 .
$$

Hence $x(t)=y(t)+R(t)$ is a positive $T$-periodic solution of System (1.1)-(1.2). The proof is complete.

REMARK 2.5. In papers $[1,12,20,23,30,39,45,50]$, the existence of positive $T$-periodic solutions of the system

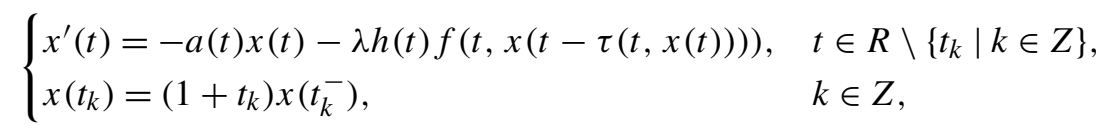

was studied under the assumptions (H3) and (H5). Hence, Theorem 2.4 generalizes the assumptions in $[1,12,20,23,30,39,45,50]$ since there is a forcing term in System (1.1)-(1.2).

Theorem 2.6. Assume that (H3)-(H5) and (H7)-(H10) hold. Then System (1.1)(1.2) has at least one positive $T$-periodic solution if $\lambda \in(A, B]$, where $A$ and $B$ are defined by

$$
\begin{aligned}
& A=\frac{2}{\sigma N} \frac{1}{D E_{-}}, \quad B=\frac{1}{D E_{+}} \min \left\{\frac{1}{M_{2}}, \frac{\sigma}{2 M}\right\}, \\
& M_{2}=\max _{(t, x) \in[0, T] \times[0,1]} g(t, x), \quad g(t, x)= \begin{cases}f(t, x), & t \in R, x \geq 0, \\
f(t, 0), & t \in R, x<0 .\end{cases}
\end{aligned}
$$


ProOF. Set $w(t)=\int_{t}^{t+T} G(t, s) h(s) d s, t \in R, z(t)=\lambda M w(t)$.

Then

$$
w(t) \leq\|w\| \leq D E_{+} .
$$

We see $z(t) \geq 0$ for all $t \geq R$ and

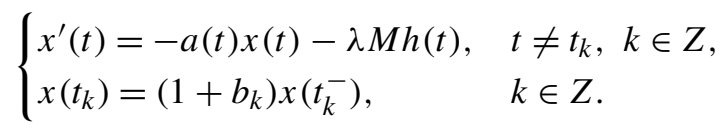

Assumption (H10) implies that System (1.1)-(1.2) has a positive $T$-periodic solution $x \in X$ if and only if $x+z=y$ is a $T$-periodic solution of

$$
\left\{\begin{aligned}
y^{\prime}(t)= & -a(t) y(t)-\lambda h(t) g(t, y(t-\tau(t, \tilde{y}(t))) & & \\
& -z(t-\tau(t, \tilde{y}(t)))), & & t \neq t_{k}, k \in Z, \\
y\left(t_{k}\right)= & \left(1+b_{k}\right) y\left(t_{k}^{-}\right), & & k \in Z,
\end{aligned}\right.
$$

and $\tilde{y}(t)=y(t)-z(t)>0$ for all $t \in R$. Define an operator $\mathbf{T}$ by

$$
\mathbf{T} y(t)=-\lambda \int_{t}^{t+T} G(t, s) h(s) g(s, y(s-\tau(s, \tilde{y}(s)))-z(s-\tau(s, \tilde{y}(s)))) d s
$$

for $y \in X$. We know, from (H3), (H4), (H5), (H8) and (H9), that $\mathbf{T}$ is completely continuous and $\mathbf{T} P \subset P$.

Let $\lambda$ be fixed with $A<\lambda \leq B$. To obtain a positive $T$-periodic solution of System (1.1)-(1.2), we perform two steps.

Step 1. Choose $\Omega_{1}=\{x \in X:\|x\|<1\}$; one has, from (H4), that

$$
y(t-\tau(t, \tilde{y}(t)))-z(t-\tau(t, \tilde{y}(t))) \leq y(t-\tau(t, \tilde{y}(t))) \leq\|y\|=1
$$

for $y \in P \cap \partial \Omega_{1}$. Then

$$
\begin{aligned}
\mathbf{T} y(t) & \leq \lambda M_{2} \int_{t}^{t+T} G(t, s) h(s) d s \\
& \leq \lambda M_{2} D E_{+} \quad(\text { using } \lambda \leq B) \\
& \leq 1=\|y\| .
\end{aligned}
$$

Then $\|\mathbf{T} y\| \leq\|y\|$ for all $y \in P \cap \partial \Omega_{1}$.

Step 2. Since $\lambda>A$, we can choose $\epsilon>0$ such that

$$
\lambda(N-\epsilon) \sigma D E_{-}>1 .
$$

Since $\lambda \leq B$, we obtain

$$
\sigma-\lambda M D E_{+} \geq \frac{\sigma}{2}
$$


For $\epsilon>0$, use (H7) and choose $\bar{R}>1$ sufficiently large that

$$
\frac{g(t, x)}{x}=\frac{f(t, x)}{x} \geq N-\epsilon \text { for }(t, x) \in[0, T] \times\left[\frac{\sigma \bar{R}}{2},+\infty\right) .
$$

Set $\Omega_{2}=\{x \in X:\|x\|<R\}$. We find, for $y \in P \cap \partial \Omega_{2}$, that

$$
\begin{aligned}
y(t)-z(t) & \geq \sigma\|y\|-\lambda M w(t) \\
& \geq \sigma \bar{R}-\lambda M D E_{+} \geq \bar{R}\left(\sigma-\lambda M D E_{+}\right) \geq \frac{\sigma \bar{R}}{2} .
\end{aligned}
$$

Hence,

$$
\begin{aligned}
\mathbf{T} y(t) & \geq-\lambda(N-\epsilon) \int_{t}^{t+T} G(t, s) h(s)(y(t-\tau(t, \tilde{y}(t)))-z(t-\tau(t, \tilde{y}(t)))) d s \\
& \geq-\frac{\lambda \sigma \bar{R}(N-\epsilon)}{2} \int_{t}^{t+T} G(t, s) h(s) d s \geq \frac{\lambda \sigma \bar{R}(N-\epsilon)}{2} D E_{-} \geq \bar{R}=\|y\| .
\end{aligned}
$$

Then $\|\mathbf{T} y\| \leq\|y\|$ for $y \in P \cap \partial \Omega_{2}$. Hence, $\mathbf{T}$ has at least one fixed point $y$ such that $1 \leq\|y\| \leq \bar{R}$. On the other hand,

$$
y(t) \geq \sigma\|y\| \geq \sigma>2 \lambda M D E_{+} \quad \text { and } \quad w(t) \leq D E_{+}<2 D E_{+}
$$

imply that $y(t)>\lambda M w(t)=z(t), t \in R$.

Hence, $x(t)=y(t)-z(t)$ is a positive $T$-periodic solution of System (1.1)-(1.2). The proof is complete.

REMARK 2.7. In [1, 12, 20, 23, 30, 39, 45, 50], the existence of positive $T$-periodic solutions of System (2.4) was studied under the assumptions (H3)' and (H5). Theorem 2.6 generalizes the assumptions seen in the literature as we allow $f$ to change sign.

Theorem 2.8. Assume that (H3)', (H4), (H5) and (H8)-(H11) hold. Then System (1.1)-(1.2) has at least one positive T-periodic solution if $\lambda \in(A, B)$, where $A$ and $B$ are defined by

$$
A=\frac{1}{\sigma l D E_{-}}, \quad B=\frac{1}{L D E_{+}} .
$$

PROOF. Define an operator $\mathbf{T}$ by

$$
\mathbf{T} y(t)=-\lambda \int_{t}^{t+T} G(t, s) h(s) f(s, y(s-\tau(s, y(s)))) d s, \quad y \in X .
$$

We see that $\mathbf{T}$ is completely continuous and $\mathbf{T} P \subset P$ because of (H4), (H5), (H8) and (H9).

Let $\lambda \in(A, B)$ be fixed. From (H11) and $\lambda<B$, we choose $\epsilon>0$ and $R_{2}>0$ such that

$$
\lambda(L+\epsilon) D E_{+} \leq 1
$$


and

$$
\frac{f(t, x)}{x} \leq L+\epsilon, \quad t \in[0, T], x \geq R_{2} .
$$

We perform two steps.

Step 1 . Set $\Omega_{1}=\left\{x \in X:\|x\|<R_{2} / \sigma\right\}$. If $y \in P \cap \partial \Omega_{1}$, then $y(t) \geq \sigma\|y\|=R_{2}$ and

$$
\begin{aligned}
\mathbf{T} y(t) & \leq-\lambda(L+\epsilon) \int_{t}^{t+T} G(t, s) h(s) y(s, \tau(s, y(s))) d s \\
& \leq-\lambda(L+\epsilon)\|y\| \int_{t}^{t+T} G(t, s) h(s) d s \leq \lambda(L+\epsilon)\|y\| D E_{+} \leq\|y\| .
\end{aligned}
$$

Then $\|\mathbf{T} y\| \leq\|y\|$ for all $y \in P \cap \partial \Omega_{1}$.

Step 2. Since $\lambda>A$, choose $\epsilon>0$ such that

$$
\sigma(l-\epsilon) D E_{-} \geq 1
$$

From (H11), choose $0<R_{1}<R_{2}$ such that

$$
\frac{f(t, x)}{x}>l-\epsilon, \quad t \in[0, T], x \in\left[0, R_{1}\right] .
$$

Let $\Omega_{2}=\left\{x \in X:\|x\|<R_{1}\right\}$. For $y \in P \cap \partial \Omega_{2}$, we find $0 \leq y(t) \leq\|y\|=R_{1}$, and

$$
\begin{aligned}
\mathbf{T} y(t) & \geq-\lambda(l-\epsilon) \int_{t}^{t+T} G(t, s) h(s) y(s, \tau(s, y(s))) d s \\
& \geq-\lambda(l-\epsilon) \int_{t}^{t+T} G(t, s) h(s) \sigma\|y\| d s \\
& \geq \lambda \sigma(l-\epsilon)\|y\| D E_{-} \geq\|y\| .
\end{aligned}
$$

Then $\|\mathbf{T} y\| \geq\|y\|$ for all $y \in P \cap \partial \Omega_{2}$. Hence, $\mathbf{T}$ has at least one fixed point $y$ such that $R_{1} \leq\|y\| \leq R_{2}$ that is a positive $T$-periodic solution of System (1.1)-(1.2). The proof is complete.

Theorem 2.9. Assume that (H3)', (H4), (H5), (H8)-(H10) and (H11)' hold. Then System (1.1)-(1.2) has at least one positive $T$-periodic solution if $\lambda \in(A, B)$, where $A$ and $B$ are defined as follows:

$$
A=\frac{1}{\sigma L^{\prime} D E_{-}}, \quad B=\frac{1}{l^{\prime} D E_{+}} .
$$

The proof is similar to that of Theorem 2.8 and is omitted.

REMARK 2.10. In [1, 12, 20, 23, 30, 39, 45, 50], the existence of positive $T$-periodic solutions of System (2.4) were studied under the assumptions that (H3) and (H5) hold and $a, h$ are nonnegative. Theorems 2.8 and 2.9 generalize these assumptions by allowing $a$ and $h$ to change sign and using $\phi(x)=1$ in these theorems reproduces the results seen in the literature. 
THEOREM 2.11. If (H3)', (H4), (H5), (H8)-(H10) hold and

$$
\lambda D E_{+} f(t, x)<\left(1-\max _{0<t_{k} \leq T} b_{k}^{-}\right) x,
$$

then System (1.1)-(1.2) has no positive T-periodic solution.

PROOF. Assume to the contrary that $y(t)$ is a positive $T$-periodic solution of System (1.1)-(1.2). We consider two cases.

CASE 1 . There is $t_{0} \in[0, T]$ such that $\|y\|=y\left(t_{0}\right)$.

For this case,

$$
\begin{aligned}
\|y\| & =y\left(t_{0}\right)=-\lambda \int_{t_{0}}^{t_{0}+T} G\left(t_{0}, s\right) h(s) f(s, y(s-\tau(s, y(s)))) d s \\
& <-\frac{1}{D E_{+}} \int_{t_{0}}^{t_{0}+T} G\left(t_{0}, s\right) h(s)\left(1-\max _{0<t_{k} \leq T} b_{k}^{-}\right) y(s-\tau(s, y(s))) d s \\
& \leq \frac{1}{D E_{+}} \cdot D E_{+}\|y\|=\|y\|,
\end{aligned}
$$

which is a contradiction.

CASE 2. We have $\|y\| \neq y(t)$ for each $t \in[0, T]$.

Then there exists $t_{k} \in[0, T]$ (an impulsive point) such that $\|y\|=y\left(t_{k}^{-} k\right)$. In this case, we have $y\left(t_{k}\right)<y\left(t_{k}^{-} k\right)$. It follows from (H8) that $-1<b_{k}<0$. So

$$
0<\frac{1-\max _{0<t_{k} \leq T} b_{k}^{-}}{1+b_{k}} \leq 1 \text {. }
$$

Since

$$
y\left(t_{k}\right)=-\lambda \int_{t_{k}}^{t_{k}+T} G\left(t_{k}, s\right) h(s) f(s, y(s-\tau(s, y(s)))) d s,
$$

we obtain

$$
\begin{aligned}
\|y\| & =y\left(t_{k} k\right)=\frac{1}{1+b_{k}} y\left(t_{k}\right) \\
& \leq-\lambda \frac{1}{1+b_{k}} \int_{t_{k}}^{t_{k}+T} G\left(t_{k}, s\right) h(s) f(s, y(s-\tau(s, y(s)))) d s \\
& <-\frac{1}{D E_{+}} \int_{t_{0}}^{t_{0}+T} G\left(t_{0}, s\right) h(s)\left(1-\max _{0<t_{k} \leq T} b_{k}^{-}\right) y(s-\tau(s, y(s))) d s \\
& \leq \frac{1}{D E_{+}} \cdot D E_{+}\|y\|=\|y\|,
\end{aligned}
$$

which is a contradiction. From Cases 1 and 2, we have that System (1.1)-(1.2) has no positive $T$-periodic solution. The proof is complete. 
THEOREM 2.12. If (H3)', (H4), (H5), (H8), (H9) and (H10) hold and

$$
\lambda \sigma D E_{-} f(t, x)>\left(1+\max _{0<t_{k} \leq T} b_{k}^{+}\right) x,
$$

then System (1.1)-(1.2) has no positive T-periodic solution.

The proof is similar to that of Theorem 2.11 and is omitted.

\section{Applications and examples}

In this section, we apply our main results to (1.3), (1.4) and (1.5) and two examples to illustrate the main results.

THEOREM 3.1. Assume that

$$
\left\{\begin{array}{l}
\mu, p, r \in X \text { with } p(t) \text { being negative and } \\
\exp \left(\int_{0}^{T} a(u) d u\right)<\prod_{0<t_{k} \leq T}\left(1+b_{k}\right), b_{k}>-1 \text { for all } k \in Z \\
\prod_{t<t_{k} \leq t+T}\left(1+b_{k}\right) \equiv \text { constant for all } t \in R .
\end{array}\right.
$$

Then (1.3) has at least one positive T-periodic solution for all $\lambda>0$.

PROOF. By choosing $f(t, x)=e^{-r(t) x}$ in System (1.1)-(1.2), we obtain (1.3). Then the proof of Theorem 3.1 follows directly from Theorem 2.8 .

THEOREM 3.2. Suppose that (3.1) holds. Then (1.4) has at least one positive $T$-periodic solution for all $\lambda>0$.

Proof. By choosing $f(t, x)=1 /\left(1+r(t) x^{n}\right)$ in System (1.1)-(1.2), we obtain (1.4). Then the proof of Theorem 3.2 follows directly from Theorem 2.8.

THEOREM 3.3. Suppose that (3.1) holds. Then there exists $\lambda^{*}>0$ such that (1.5) has at least one positive $T$-periodic solution if $\lambda>\lambda^{*}$ and has no positive $T$-periodic solution if $0<\lambda<\lambda^{*}$.

Proof. Choose $f(t, x)=x e^{-r(t) x}$ in System (1.1)-(1.2). Then $l=1$ and $L=0$, as defined in (H11).

First, by Theorem 2.8, we know that there are $A>0$ such that (1.5) has at least one positive $T$-periodic solution if $\lambda>A$. 
Second, if (1.5) has a positive $T$-periodic solution $N(t)$, then

$$
\begin{aligned}
N(t) & =-\lambda \int_{t}^{t+T} G(t, s) p(s) N(s-\tau(s)) e^{-r(s) N(s-\tau(s))} d s \\
& <-\lambda \int_{t}^{t+T} G(t, s) p(s) N(s-\tau(s)) d s \\
& \leq-\lambda\|N\| \int_{t}^{t+T} G(t, s) p(s) d s \\
& \leq \lambda\|N\| D \prod_{0<t_{k} \leq T}\left(1+b_{k}^{+}\right) \exp \left(\int_{0}^{T} a^{+}(u) d u\right) \int_{0}^{T} p(s) d s .
\end{aligned}
$$

It follows that

$$
\|N\| \leq \lambda\|N\| D \prod_{0<t_{k} \leq T}\left(1+b_{k}^{+}\right) \exp \left(\int_{0}^{T} a^{+}(u) d u\right) \int_{0}^{T} p(s) d s .
$$

Thus,

$$
\lambda>\frac{1}{D \prod_{0<t_{k} \leq T}\left(1+b_{k}^{+}\right) \exp \left(\int_{0}^{T} a^{+}(u) d u\right) \int_{0}^{T} p(s) d s} .
$$

Hence, there exists $B>0$ such that (1.5) has no positive $T$-periodic solution if $\lambda \leq B$.

Finally, it is easy to show that if $\lambda_{0}>0$ satisfies

$$
\begin{cases}N^{\prime}(t)=-\mu(t) N(t)+\lambda_{0} p(t) N(t-\tau(t)) e^{-r(t) N(t-\tau(t))}, & t \neq t_{k}, k \in Z, \\ N\left(t_{k}\right)=\left(1+b_{k}\right) N\left(t_{k}^{-}\right), & k \in Z,\end{cases}
$$

has positive $T$-periodic solution, then

$$
\begin{cases}N^{\prime}(t)=-\mu(t) N(t)+\lambda p(t) N(t-\tau(t)) e^{-r(t) N(t-\tau(t))}, & t \neq t_{k}, k \in Z, \\ N\left(t_{k}\right)=\left(1+b_{k}\right) N\left(t_{k}^{-}\right), & k \in Z\end{cases}
$$

has at least one positive $T$-periodic solution for all $\lambda>\lambda_{0}$.

Together with above discussions, this completes the proof.

OPEN PROBLEM. Under the assumptions in Theorem 3.3, does (1.5) posses a positive $T$-periodic solution if $\lambda=\lambda^{*}$ ?

Now, we present two examples to illustrate the main results.

EXAMPLE 3.4. Consider the following impulsive differential equation

$$
\begin{cases}x^{\prime}(t)+[\sin 2 \pi t+2] x(t)=-\lambda[1+\cos 2 \pi t][|x(t)|-2], & t \neq t_{k}, k \in Z, \\ x\left(t_{k}\right)=\left(1+b_{k}\right) x\left(t_{k}^{-}\right), & k \in Z\end{cases}
$$

where $\lambda>0$ is a parameter, $b_{k}>-1$ for all $k \in Z$ satisfying (H8). 
Returning to System (1.1)-(1.2), let $f(x)=|x|-2, \quad h(t)=1+\cos (2 \pi t)$, $a(t)=2+\sin (2 \pi t)$. Choose $M=2$ and the period $T=1$. It is easy to see that $N=1$ and the assumptions (H3)-(H5) and (H7)-(H10) hold.

One can find that $M_{2}=1 ; g(t, x)=x^{2}$ and

$$
A=\frac{2}{e^{-2}} \frac{-e^{-2} \prod_{0<t_{k} \leq 1}\left(1+b_{k}\right)}{\prod_{0<t_{k} \leq 1}\left(1-b_{k}^{-}\right)} \text {and } B=\frac{-e^{-2} \prod_{0<t_{k} \leq 1}\left(1+b_{k}\right)}{\prod_{0<t_{k} \leq 1}\left(1+b_{k}^{+}\right)} \min \left\{\frac{1}{e^{2}}, \frac{e^{-2}}{4 e^{2}}\right\} \text {. }
$$

It follows from Theorem 2.6 that (3.2) has at least one positive periodic solution if $\lambda \in(A, B]$.

REMARK 3.5. Since the nonlinearity in (3.2) is $f(t, x)=|x|-2$ that changes sign on $[0,+\infty)$, the results in $[1,12,20,23,30,39,45,50,53]$ cannot be applied on (3.2).

EXAMPLE 3.6. Consider the delay differential equation without impulse effects

$$
x^{\prime}(t)+a(t) x(t)=-\lambda h(t) x(t-\tau)+r(t), \quad t \in R,
$$

where $a, h$ and $r$ are $T$-periodic continuous functions with $\int_{0}^{T} a(s) d s<0, \lambda>0$ a constant.

Returning to System (1.1)-(1.2), choose $f(t, x)=x, b_{k}=0$ for all $k \in Z$.

In (3.3), we find that $N=1$, as defined in (H7), and

$$
\begin{gathered}
A=\frac{2-2 e^{\int_{0}^{T} a(s) d s}}{e^{-\int_{0}^{T}|a(s)| d s} \int_{0}^{T} h(s) d s}, \quad R(t)=\int_{t}^{t+T} \frac{e^{\int_{t}^{s} a(u) d u}}{1-e^{\int_{0}^{T} a(u) d u}} r(s) d s, \\
R_{0}=\|R\|, \quad M_{2}=\frac{R_{0}(\sigma+1)}{\sigma} \text { and } B=\frac{\sigma-\sigma e^{\int_{0}^{T} a(u) d u}}{(\sigma+1) e^{-\int_{0}^{T} a^{-}(u) d u} \int_{0}^{T} h(s) d s} .
\end{gathered}
$$

It is easy to see that assumptions (H3)', (H4), (H5) and (H7) hold. It follows from Theorem 2.4 that (3.3) has at least one positive $T$-periodic solution for all $\lambda \in(A, B]$.

REMARK 3.7. The authors in [3, 13, 52] studied the existence of positive $T$-periodic solutions of the following functional differential equation

$$
y^{\prime}(t)=-a(t) y(t)+\lambda h(t) f(y(t-\tau(t))),
$$

where $a=a(t), h=h(t)$ and $\tau=\tau(t)$ are continuous $T$-periodic functions, $T>0$, $\lambda>0$, that $a=a(t), f=f(t)$ and $h=h(t)$ are nonnegative, and that $a\left(t_{0}\right)>0$ for some $t_{0} \in[0, T]$. One can see that the results in $[12,14,32,52]$ cannot be applied to $(3.3)$.

\section{Acknowledgements}

The author is very grateful to two anonymous referees and the editors for their careful reading of the paper and constructive comments which helped to improve the presentation of the results. 


\section{References}

[1] M. Bartha, "Periodic solutions for differential equations with state-dependent delay and positive feedback", Nonlinear Anal. 53 (2003) 839-857.

[2] L. Chen and J. Sun, "Nonlinear boundary value problem of first order impulsive functional differential equations", J. Math. Anal. Appl. 318 (2006) 726-741.

[3] S. Cheng and G. Zhang, "Existence of positive periodic solutions for non-autonomous functional differential equations", Electron. J. Differential Equations 59 (2001) 1-8.

[4] M. Choisy, J. Guegan and P. Rohani, "Dynamics of infectious diseases and pulse vaccination: teasing apart the embedded resonance effects", Physica D 22 (2006) 26-35.

[5] W. Ding, J. Mi and M. Han, "Periodic boundary value problems for the first order impulsive functional differential equations", Appl. Math. Comput. 165 (2005) 433-446.

[6] A. d'Onofrio, "On pulse vaccination strategy in the SIR epidemic model with vertical transmission", Appl. Math. Lett. 18 (2005) 729-732.

[7] A. d'Onofrio, "A general framework for modeling tumor-immune system competition and immunotherapy: mathematical analysis and biomedical inferences", Physica D 208 (2005) 220-235.

[8] S. Gao, L. Chen, J. Nieto and A. Torres, "Analysis of a delayed epidemic model with pulse vaccination and saturation incidence", Vaccine 24 (2006) 6037-6045.

[9] W. Gurney, S. Blythe and R. Nisbet, "Nicholson blowflies revised", Nature 287 (1980) 17-21.

[10] J. Hale and S. Lonel, Introduction to functional differential equations (Springer, Berlin, 1993).

[11] D. Jiang, J. Nieto and W. Zuo, "On monotone method for first and second order periodic boundary value problems and periodic solutions of functional differential equations", J. Math. Anal. Appl. 289 (2004) 691-699.

[12] S. Kang and G. Zhang, "Existence of nontrivial periodic solutions for first order functional differential equations", Appl. Math. Lett. 18 (2005) 101-107.

[13] Y. Kuang and H. Smith, "Slowly oscillating periodic solutions of nonautonomous state-dependent delay equations", Nonlinear Anal. 19 (1992) 855-872.

[14] V. Lakshmikantham, D. Bainov and P. Simenov, Theory of impulsive differential equations (World Scientific, Singapore, 1989).

[15] W. Li and H. Huo, "Existence and global attractivity of positive periodic solutions of functional differential equations with impulses", Nonlinear Anal. 59 (2004) 857-877.

[16] W. Li and H. Huo, "Global attractivity of positive periodic solutions for an impulsive delay periodic model of respiratory dynamics", J. Comput. Appl. Math. 174 (2005) 227-238.

[17] X. Li, X. Lin, D. Jiang and X. Zhang, "Existence and multiplicity of positive periodic solutions to functional differential equations with impulse effects", Nonlinear Anal. 62 (2005) 683-701.

[18] J. Li, J. Nieto and J. Shen, "Impulsive periodic boundary value problems of first order differential equations", J. Math. Anal. Appl. 325 (2007) 226-236.

[19] J. Li and J. Shen, "Existence of positive periodic solutions to a class of functional differential equations with impulses", Math. Appl. (Wuhan) 17 (2004) 456-463.

[20] J. Li and J. Shen, "Periodic boundary value problems for delay differential equations with impulses", J. Comput. Appl. Math. 193 (2006) 563-573.

[21] W. Li and Z. Wang, "Existence and global attractivity of positive periodic solutions for the impulsive delay Nicholson's blowflies model”, J. Comput. Appl. Math. 50 (2005) 41-47.

[22] X. Li, X. Zhang and D. Jiang, "A new existence theory for positive periodic solutions to functional differential equations with impulse effects", Comput. Math. Appl. 51 (2006) 1761-1772.

[23] Y. Li and L. Zhu, "Positive periodic solutions for a class of higher-dimensional state-dependent delay functional differential equations with feedback control", Appl. Math. Comput. 159 (2004) 783-795.

[24] R. Liang and J. Shen, "Periodic boundary value problem for the first order impulsive functional differential equations", J. Comput. Appl. Math. 202 (2007) 498-510.

[25] Y. Liu, "Further results on periodic boundary value problems for nonlinear first order impulsive functional differential equations", J. Math. Anal. Appl. 327 (2007) 435-452. 
[26] Y. Liu, Z. Bai and W. Ge, "Positive periodic solutions of impulsive delay differential equations with sign-changing coefficients", Port. Math. 61 (2004) 177-192.

[27] Y. Liu and W. Ge, "Asymptotic behavior of certain delay differential equations with forcing term", J. Math. Anal. Appl. 280 (2003) 350-363.

[28] Y. Liu and W. Ge, "Global attractivity in delay "food-limited" models with exponential impulses", J. Math. Anal. Appl. 287 (2003) 200-216.

[29] Y. Liu and W. Ge, "Positive periodic solutions of state-dependent functional differential equations", Appl. Anal. 84 (2005) 1079-1094.

[30] P. Liu and Y. Li, "Positive periodic solutions of infinite delay functional differential equations depending on a parameter", Appl. Math. Comput. 150 (2004) 159-168.

[31] Y. Liu, J. Xia and W. Ge, "Positive periodic solutions of impulsive functional differential equations", J. Appl. Math. Comput. 19 (2005) 261-280.

[32] G. Liu, J. Yan and F. Zhang, "Existence and global attractivity of unique positive periodic solution for a model of hematopoiesis", J. Math. Anal. Appl. 334 (2007) 157-171.

[33] Y. Liu and B. Zhang, "Global attractivity of a class of delay differential equations with impulses", ANZIAM J. 45 (2003) 271-284.

[34] M. Mackey and I. Glass, "Oscillations and chaos in physiological control systems", Science 197 (1977) 287-289.

[35] J. Nieto and R. Rodriguez-Lopez, "Remarks on periodic boundary value problems for functional differential equations", J. Comput. Appl. Math. 158 (2003) 339-353.

[36] J. Nieto and R. Rodriguez-Lopez, "Monotone method for first-order functional differential equations", Comput. Math. Appl. 52 (2006) 471-484.

[37] J. Nieto and R. Rodriguez-Lopez, "Periodic boundary value problem for non-Lipschitzian impulsive functional differential equations", J. Math. Anal. Appl. 318 (2006) 593-610.

[38] J. Nieto and R. Rodriguez-Lopez, "New comparison results for impulsive integro-differential equations and applications", J. Math. Anal. Appl. 328 (2007) 1343-1368.

[39] E. Pielou, Mathematical ecology (Wiley Interscience, New York, 1977).

[40] D. Qian and X. Li, "Periodic solutions for ordinary differential equations with sublinear impulsive effects", J. Math. Anal. Appl. 303 (2004) 288-303.

[41] G. Rost, "On the global attractivity controversy for a delay model of hematopoiesis", Appl. Math. Comput. 190 (2007) 846-850.

[42] S. Saker and S. Agarwal, "Oscillatory and global attractivity in a periodic Nicholson's blowflies model", Math. Comput. Modelling 35 (2002) 719-731.

[43] S. Tang and L. Chen, "Density-dependent birth rate, birth pulses and their population dynamic consequences", J. Math. Biol. 44 (2002) 185-199.

[44] S. Tang and L. Chen, "Global attractivity in a "food-limited" population model with impulsive effects", J. Math. Anal. Appl. 292 (2004) 211-221.

[45] H. Wang, "Positive periodic solutions of functional differential equations", J. Differential Equations 202 (2004) 354-366.

[46] W. Wang, H. Wang and Z. Li, "The dynamic complexity of a three-species Beddington-type food chain with impulsive control strategy", Chaos Solitons Fractals 32 (2007) 1772-1785.

[47] M. Wazewska-Czyzewska and A. Lasota, "Mathematical models of red cells system", Mat. Stosow. 6 (1976) 25-40.

[48] P. Weng and M. Liang, "The existence and behavior of periodic solutions of a Hematopoiesis model", Math. Appl. 8 (1995) 434-439.

[49] J. Yan, "Existence and global attractivity of positive periodic solution for an impulsive LasotaWazewska model", J. Math. Anal. Appl. 279 (2003) 111-120.

[50] J. Yan, "Existence of positive periodic solutions of impulsive functional differential equations with two parameters", J. Math. Anal. Appl. 327 (2007) 854-868.

[51] J. Yan, A. Zhao and J. Nieto, "Existence and global attractivity of positive periodic solution of periodic single species impulsive Lotka-Volterra systems", Math. Comput. Modelling 40 (2004) $509-518$. 
[52] G. Zhang and S. Cheng, "Positive periodic solutions of nonautonomous functional differential equations depend on a parameter", Abstr. Anal. Appl. 7 (2002) 279-286.

[53] W. Zhang and M. Fan, "Periodicity in a generalized ecological competition system governed by impulsive differential equations with delays", Math. Comput. Modelling 39 (2004) 479-493.

[54] X. Zhang, Z. Shuai and K. Wang, "Optimal impulsive harvesting policy for single population", Nonlinear Anal. Real World Appl. 4 (2003) 639-651. 